\title{
Design considerations for a theory-driven exergame-based rehabilitation program to improve walking of persons with stroke
}

\author{
Seline Wüest • Rolf van de Langenberg • \\ Eling D. de Bruin
}

Received: 1 August 2013 / Accepted: 20 November 2013 / Published online: 7 December 2013

(C) The Author(s) 2013. This article is published with open access at Springerlink.com

\begin{abstract}
Virtual rehabilitation approaches for promoting motor recovery has attracted considerable attention in recent years. It appears to be a useful tool to provide beneficial and motivational rehabilitation conditions. Following a stroke, hemiparesis is one of the most disabling impairments and, therefore, many affected people often show substantial deficits in walking abilities. Hence, one of the major goals of stroke rehabilitation is to improve patients' gait characteristics and hence to regain their highest possible level of walking ability. Because previous studies indicate a relationship between walking and balance ability, this article proposes a stroke rehabilitation program that targets balance impairments to improve walking in stroke survivors. Most currently, available stroke rehabilitation programs lack a theory-driven, feasible template consistent with widely accepted motor learning principles and theories in rehabilitation. To address this hiatus, we explore the potential of a set of virtual reality games
\end{abstract}

\footnotetext{
Authors' contributions SW performed literature review and design considerations and contributed to writing the manuscript. RvdL performed design considerations and contributed to writing the manuscript. EdB initiated the study, assisted in both literature review and design considerations and contributed to writing the manuscript. All authors read and approved the final manuscript.

The authors declare that the submitted paper, the data, and the results have not been published anywhere before.
}

S. Wüest $(\bowtie) \cdot R$. van de Langenberg $\cdot$ E. D. de Bruin Department of Health Sciences and Technology, Institute of Human Movement Sciences and Sport, Wolfgang-Pauli-Str. 27, 8093 Zurich, Switzerland

e-mail: seline.wueest@hest.ethz.ch

R. van de Langenberg

e-mail: rvdlangenberg@ethz.ch

E. D. de Bruin

e-mail: eling.debruin@hest.ethz.ch

S. Wüest $\cdot$ R. van de Langenberg $\cdot$ E. D. de Bruin

ETH Zurich, Zurich, Switzerland specifically developed for stroke rehabilitation and ordered according to an established two-dimensional motor skill classification taxonomy. We argue that the ensuing "exergame"based rehabilitation program warrants individually tailored balance progression in a learning environment that allows variable practice and hence optimizes the recovery of walking ability.

Keywords Strokerehabilitation · Gentile's taxonomy · Virtual reality $\cdot$ Exergames $\cdot$ Motor learning

\section{Introduction}

Virtual reality technique can be combined with targeted exergames development with the aim to promote motor rehabilitation. Recently, a number of researchers have pointed to the potential of virtual reality applications in health care $[6,20$, $28,36,48,50]$ and hence sparked the introduction of this technology within rehabilitation medicine. The use of socalled "exergames"- virtual reality games that involve physical exercise - has been proposed as a valuable instrument to encourage participation in rehabilitation and improve the adherence to therapy programs because of engaging the user [6, 12, 48]. For example, a study by Rizzo and Kim [48] demonstrated that virtual reality-based games reduce patients' dreariness and simultaneously increase their motivation for rehabilitation practice. Accordingly, virtual reality provides the capacity to simulate scenarios that are effective in attracting the performers' attention. Simulated circumstances can be used to elicit a thrilling ambience, whereas the patient can still perform movement and behaviors in a safe and controlled environment.

For best results in rehabilitation, video games specifically designed for therapy should be used [5]. Because conventional video games were primarily developed for entertainment 
purposes [63], most of these are not practical for rehabilitation [5]. One hurdle facing the successful use of exergames in rehabilitation is that many off-the-shelf video games are too complex for use by functionally impaired persons or elderly people [12]. Video games must therefore be developed to take into consideration the cognitive and physical limitations, as well as the interest sets, of the trainees [12]. To date, however, the vast majority of research has focused on games developed for the entertainment market which fails to adapt the gameplay according to patients' rehabilitation requirements [44].

FP7 is the short name for the Seventh Framework Programme for Research and Technological Development, the European Union's main instrument for funding research in Europe running from 2007 to 2013 . FP7 is also designed to respond to Europe's employment needs, competitiveness, and quality of life [c.f. http://ec.europa.eu/research/fp7/index_en. $\mathrm{cfm}$ ?pg=understanding]. The research project "Rehabilitative Wayout In Responsive home Environments" (REWIRE) [46], which is funded by FP7, aims to develop, integrate, and field test an innovative virtual reality-based rehabilitation platform for people with stroke. The platform should allow patients, discharged from the hospital, to continue intensive rehabilitation at home under remote monitoring by the hospital. The main idea is to combine off-the-shelf components (e.g., the tracking device Microsoft Kinect [30], the force plate Tymo [58]) in a robust and reliable way and render a system that can be used in the stroke patients' homes. In the context of the REWIRE project, the need has been recognized for exergames specifically targeted at walking rehabilitation [5].

When creating a virtual reality-based stroke treatment approach, the development of suitable exergames is undoubtedly an important requirement. There have been, to date, some studies that deal with the development of video games adequate for therapy purposes. Based on specific game design principles, they intend to provide engaging and challenging treatment conditions to achieve a high level of motivation for rehabilitation practicing [1, $6,7,24,33,43,59]$. Moreover, it is important that the developed exergames are functionally integrated in a wellelaborated therapy program. On the one hand, the rehabilitation program should be aimed at appropriate rehabilitation goals. That is, it should be aimed at those goals that are considered to be essential for (and by) stroke victims. On the other hand, the rehabilitation program should be consistent with established training principles to successfully reach these goals. In this article, we describe how tailored exergames for stroke patients can be developed based on a theoretical framework. We will discuss key exergame design and training program content considerations for patients with stroke, which may be theoretically linked to impaired walking performance due to the stroke event.

\section{Stroke-induced motor impairments and their treatment approach}

Worldwide, there are about 4.8 million survivors of stroke of whom about 1.1 million suffer lasting functional disabilities [14]. The specific disabilities caused by stroke vary greatly depending on the brain area that is damaged. Hemiparesis - a paralysis that characteristically affects an arm and leg on one side of the body - is one of the most common stroke-induced impairments [34] and often leads to a number of negative walking-related consequences. The typical "hemiparetic gait" post-stroke is associated with a reduced walking velocity, cadence and stride length, with gait asymmetry, and with a prolonged double-support and stance-phase duration of both lower extremities $[15,26,27,45,61]$. It is for this reason that many stroke victims show marked deficits in walking abilities [51]. There is general agreement that an association exists between the degree of independent mobility and quality of life $[47,54,56]$. Consequently, a central goal of stroke rehabilitation should be to improve gait characteristics and to retrain the patient to the highest possible level of walking ability [16, 34]. The findings demonstrated by Vincent et al. [60] confirmed the importance of enhancing stroke patients' functional mobility through rehabilitation. Vincent and colleagues [60] set out to reveal rehabilitation needs from the perspective of four different parties involved in the stroke rehabilitation process (stroke patients, caregivers, health professionals, and healthcare managers) so as to better plan the post-stroke treatment service. According to patients' statements - which, in our view, should have high priority when designing rehabilitation programs - continued rehabilitation should focus primarily on motor activities, such as walking. Patients' statements thus further highlight the importance of gait recovery.

Good balance skills are an important determinant of walking performance and impaired balance ability is assumed to be related to a decreased locomotor function $[18,62]$. Considering that hemiparesis not only affects gait characteristics, but also often leads to diminished balance skills, a post-stroke rehabilitation program targeting deficits in static and dynamic balance may be an effective way to restore independent functional walking. In recent years, another factor contributing to walking recovery has been suggested: Several authors noted that task-related activities lead to greater improvements in post-stroke walking competency than non-task-related practices [13, 42, 49]. Specifically, they suggested that intervention protocols that include actual walking tasks improve walking skills to a greater extent than rehabilitation programs that do not. Additionally, there is robust evidence in the motor learning literature that the best outcomes in terms of long-term retention and transfer of skills are achieved when principles of motor learning are integrated into treatment protocols. In rehabilitation, it is widely accepted that more practice is better 
and that an intense structured therapy program with numerous repetitions of various, challenging tasks supports motor skill acquisition [38]. Specifically, two important elements in our consideration are the motor learning principles variable practice and progression. These principles are known to positively affect gait rehabilitation and, hence, should be considered in developing a rehabilitation program.

The literature to date has found that variable practice leads to better transfer and retention of motor skills than if a constant practice structure is used $[22,23,31,38,52]$. Instead of performing one task repeatedly and always in the same manner (constant practice), a specific task should be practiced differently throughout a treatment session by varying the conditions of practice (variable practice) [38]. Krakauer [31] - in his contribution published in the Current Opinion in Neurology - stated that variable practice is a fundamental principle in terms of retaining learning over time and that a consistent agreement in the literature exists indicating that varied practice is superior to repetitive identical tasks when it comes to motor learning. The principle of progression holds that motor learning and rehabilitation programs benefit from a continuous adaptation of task difficulty to increasing skill level [19]. For successful learning, an optimal challenging training situation should be given providing an appropriate task difficulty level according to individuals' capacities [17]. Furthermore, it can be hypothesized that the training effects will be enhanced when the locomotor practice gets combined with direct feedback; e.g., visual feedback. Visual feedback can be used to provide information about a patient's movement or the result of a movement and is known to promote postural control and stability [2, 19, 25, 53, 62].

From the above, it follows that rehabilitation programs for stroke survivors are well-advised to train balance and walking skills by means of a variety of balance- and walking-specific exercises, the difficulty of which is progressively adapted to patients' skill level, in combination with direct visual feedback on performance.

Although previous studies have emphasized the importance of theory-based practice and rehabilitation $[3,11,37$, 57], there is still a lack of established concepts underlying the practical implementation of motor skill learning principles and theories in rehabilitation. A desirable template would be one that is simple and feasible and that facilitates the taskspecific, progressive, and variable training of balance and walking skills. The motor skill taxonomy proposed by Gentile [21] seems to constitute just such a template for rehabilitation programs because it provides a twodimensional basis for classifying a variety of motor skills. Based on the above, the aim of this article is to develop and describe a tailored exergames-based stroke rehabilitation program that is based on a theoretical framework.

\section{Methods}

Four prevalent classification systems exist for identifying common characteristics of motor skills. Three of these categorize motor skills according to one common characteristic of the skill and lead to one-dimension classification systems. In contrast, Gentile's taxonomy considers two general skill characteristics and offers, therefore, a broader concept leading to a twodimensional classification system of motor skills. To highlight the high potential of Gentile's two-dimensional approach, it is worthwhile to focus at first on the one-dimensional classification systems and discus some of their limitations:

A description of these classification systems is given by Magill [35] (pp. 5-16). One one-dimension classification system differentiates skills depending on the sizes of the primary muscle groups required to produce an action (gross motor skills vs. fine motor skills). A second one-dimensional system considers the specificity of a movement's beginning and end points to categorize motor skills (continuous motor skills vs. discrete motor skills). The third one-dimension classification system makes a distinction according to the stability of the environmental context in which an action is being performed (open motor skills vs. closed motor skills) [35, 39]. These onedimensional classification systems raise the problem that they fail to capture the complexity of many motor skills [35] by only focusing on a single aspect of motor skills.

\section{Gentile's motor skill taxonomy}

Gentile presented a systematic classification system to categorize motor skills and movement according to two general dimensions of physical actions [32]. The first dimension, environmental context, refers to the environmental conditions to which the performer has to react in order to successfully perform a task. This dimension is characterized by two indicators: (a) regulatory conditions and (b) intertrial variability. The regulatory conditions indicate relevant environmental features that constrain movement execution and may either be stationary (stationary regulatory conditions) or moving (in-motion regulatory conditions). With the indicator intertrial variability, Gentile's taxonomy differentiates between regulatory conditions that change between trials (intertrial variability) and those that do not (no intertrial variability). The second dimension, action function, is also characterized by two indicators: (a) body orientation and (b) object manipulation. Body orientation indicates whether an action requires the performer to move from one location to another (body transport) or not (body stability). Object manipulation indicates whether an object has to be controlled during the action performance (object manipulation) or not (no object manipulation).

Through the interaction of the resulting four environmental context characteristics and four action function characteristics, 
Gentile defines 16 different motor skill categories that provide a comprehensive template to classify motor skills (Table 1). The taxonomy is a good means of becoming aware of the skill characteristics that make skills distinct from, as well as related to, other skills, and is an excellent guide for establishing practice or training routines [35].

According to Gentile, the easiest skill category can be found at the top left position (1A). Moving either rightward or downward in the table renders the skill category more difficult, so that the most difficult skill category can be found at the bottom right of the table (4D). For the action function dimension, this implies that body transport is more difficult than body stability and object manipulation more difficult than no object manipulation. Importantly, it also assumes a hierarchy in the action function characteristics: skills involving body transport but no object manipulation are more difficult than those involving object manipulations but not body transport. For the environmental context dimension, the same pattern of progression is assumed.

Thus, Gentile's taxonomy allows a systematic progression in difficulty of motor tasks and meets the demand of the motor learning principle progression. Moreover, each of the 16 categories is associated with unique features based on the twodimensional approach and, consequently, the taxonomy involves task variations. Obviously, Gentile's classification system is consistent with the motor learning principle variable practice.

There are several reasons for assuming that this taxonomy provides a valuable tool for developing a theory-based rehabilitation program using virtual reality. Considering the explanations above, it is evident that Gentile's taxonomy is in accordance with the generally accepted motor learning principles progression and variable practice. The taxonomy provides a well-structured framework and the $4 \times 4$ table can serve as a useful guide for preparing a systematically coherent motor

Table 1 Gentile's taxonomy of motor skills [35]

\begin{tabular}{|c|c|c|c|c|c|}
\hline & & \multicolumn{4}{|c|}{ Action function } \\
\hline & & \multicolumn{2}{|c|}{ Body stability } & \multicolumn{2}{|c|}{ Body transport } \\
\hline \multicolumn{2}{|c|}{$\begin{array}{c}\text { Environmental } \\
\text { context }\end{array}$} & $\begin{array}{c}\text { No object } \\
\text { manipulation }\end{array}$ & $\begin{array}{c}\text { Object } \\
\text { manipulation }\end{array}$ & $\begin{array}{c}\text { No object } \\
\text { manipulation }\end{array}$ & $\begin{array}{c}\text { Object } \\
\text { manipulation }\end{array}$ \\
\hline 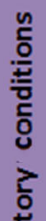 & 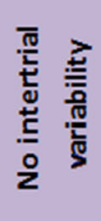 & $1 A$ & $1 B$ & $1 \mathrm{C}$ & 1D \\
\hline 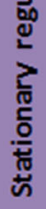 & 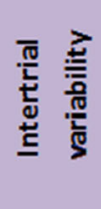 & $2 A$ & $2 B$ & $2 \mathrm{C}$ & 2D \\
\hline 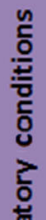 & 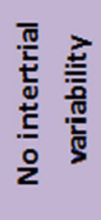 & $3 A$ & 3B & $3 C$ & 3D \\
\hline 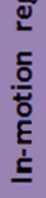 & 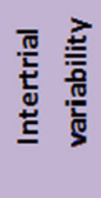 & $4 A$ & $4 B$ & $4 C$ & 4D \\
\hline
\end{tabular}


learning concept in a simple manner. For implementing Gentile's taxonomy-based approach, exergames might demonstrate an optimal opportunity for providing beneficial treatment conditions. On the one hand, as we referred above, there is high potential for creating a motivating rehabilitation environment using virtual reality. On the other hand, the taxonomy seems suited for virtual reality applications where the environment, task complexity, and other contextual factors related to exercise performance can be manipulated. Exergame parameters can be easily modified and, thereby, adjusted to the demands of the taxonomy-included skill categories.

\section{Gentile's taxonomy-based exergames to improve stroke patients' balance and walking skills}

To provide a post-stroke rehabilitation program focused on improved balance skills in standing and walking in accordance with Gentile's framework, we designed six basic exergames. Based on these six basic exergames, we created 16 virtual reality scenarios, either by making game parameter modifications or by substituting another basic game. Each scenario corresponds to one of the 16 skill categories included in the taxonomy (Table 2).

In accordance with a phased iterative approach suggested by Campbell et al. [8], this paper demonstrates the theoretical phase - the first step - when developing and evaluating complex research-based interventions to improve health. Thus, a set of six basic exergames appears to be appropriate in the research process of designing an exergame-based stroke rehabilitation program. It keeps the number of games that have to be developed manageable and - with the same game being implemented across adjacent skill categories - it renders systematic progression more straightforward.

Below, we will briefly describe the content of each of the 16 skill categories.

Table 2 Exergame scenarios corresponding to Gentile's skill categories

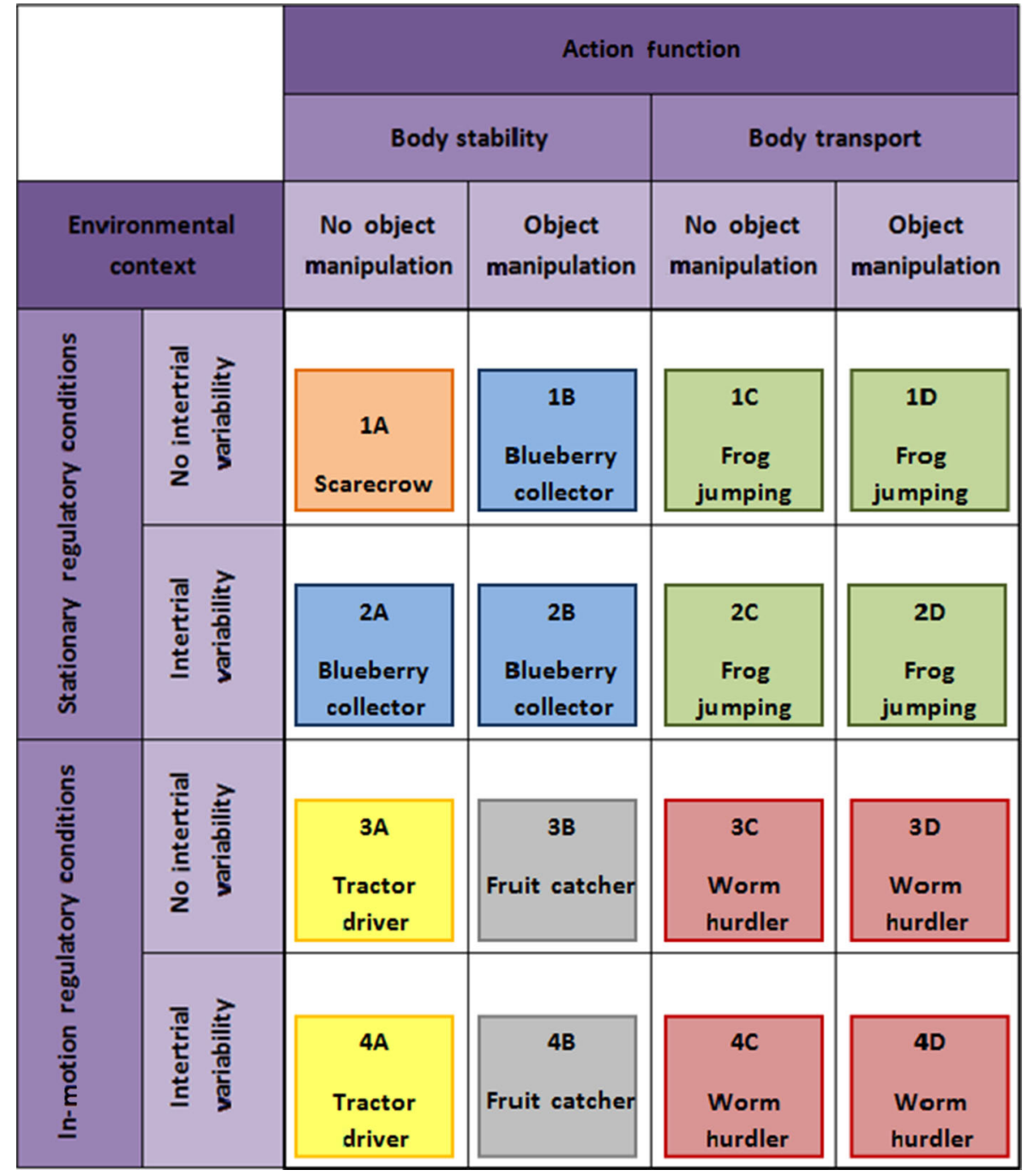


1A The first phase of the rehabilitation program focuses on the basic physical skill of standing quietly. In this exergame, the patient is represented as a scarecrow avatar and is required to maintain a cursor indicating the body's center of pressure (COP) within a predefined marked circle on a computer screen [40]. The exergame focuses on stance steadiness (sway) that demonstrates a relevant aspect related to an individual's balance ability [40]. Due to hemiparesis after stroke, patients often show an increased amount of postural sway [40].

1B Whereas the game Scarecrow described for skill category $1 \mathrm{~A}$ demands a centered and stable COP position, this game (called Blueberry Collector) uses controlled COP displacements for successful performance. This exergame targets stroke patients' dynamic stability, because subjects with hemiparesis often suffer from reduced limits of stability [10]. The virtual environment represents a field of blueberries, which have to be collected by a virtual farmer. The farmer's moving is controlled by the patient's weight shifting tasks and, in this way, the blueberries can be gathered. Because the position of the berries in the field is fixed and unvarying between the different exergame trials, stationary regulatory conditions are given and no intertrial variability. To meet the requirement of object manipulation, a virtual basked filled with blueberries is placed on the farmer's head. In order to prevent berries from falling down, the patient must adopt an ideal upright body posture.

1C Previous exercise interventions have shown that stepping exercises indicate an effective treatment method to improve postural stability and balance $[9,29,41]$, Accordingly, this exergame (called Frog jumping) requires the control of the COP displacements during stepping movements. The virtual environment represents a pond with several stones placed in it. By doing steps in a specific direction, the patient can control a frog jumping from one stone to another. A virtual arrow indicates which particular stone has to be targeted by the patient. In accordance with the taxonomy conditions, the position of each stone within the pond is fixed (stationary regulatory conditions) and the order of the targeted stones unchangeable from one exergame trial to the next (no intertrial variability).

1D According to Gentile's taxonomy, the skill category 1D can be seen as a progression of 1C. Thus, the game Frog jumping described for $1 \mathrm{C}$ can be extended by integrating an object manipulation task. Specifically, in this game, the virtual frog is wearing a crown and it is the patient's task to perform stepping tasks with an upright body position in order to keep the virtual crown correctly positioned.

2A The game Blueberry collector described above is not only adequate for skill category $1 \mathrm{~B}$, but also for category
2A by making small adaptations. On the one hand, the virtual basket on the farmer's head has to be removed to get the condition no object manipulation. On the other hand, because intertrial variability is required, the position of the berries varies within exergame trials.

2B For skill category 2B, the exergame Blueberry collector for category $2 \mathrm{~A}$ can be extended with an object manipulation task. Thus, the virtual farmer is balancing a basket on his head such as in the exergame described for skill category $1 \mathrm{~B}$.

2C We use the game Frog jumping described for category $1 \mathrm{C}$ with the only modification of providing a random order of targeted stones - marked by the arrow - to realize intertrial variability. Consequently, each time when the game has been played, a unique step performance pattern is required by the patient.

2D To meet the taxonomy requirements in skill category 2D, the game Frog jumping of category 2C can easily be adapted. The only modification that has to be done concerns the need for object manipulation. Hence, in this game, the frog is wearing a crown for which the patient has to control his/her position while playing the game.

3A For skill category $3 \mathrm{~A}$, we created a game called Tractor driver. The exergame presents a moving tractor for which the driving speed is defaulted by the video game. The direction of driving is controlled by the patient's weight shifting movements. When playing this exergame, the ability to move the COP in a standing posture without loss of balance (dynamic stability) is being trained [40]. Specifically, the purpose of this game is to fork up hay bales through directing the moving tractor from one hay bale to another. The hay bales are widely spread over the soil. Considering that the speed of the moving tractor is default and cannot be manipulated by the patient themselves, in-motion regulatory conditions are given. There is a fixed position of each hay bale unchangeable from one exergame trial to the next to ensure no intertrial variability.

3B In this exergame (called Fruit catcher), the patient is represented as an avatar balancing a fruit basket on the head. Virtual apples are falling down from a tree (inmotion regulatory conditions) that should be caught by the basket. The patient has to perform target-oriented COP shifts to get an optimal avatar position and the apples are falling into the basket. This exergame focuses on dynamic stability due to controlled weight shifting movements to selected targets. For presenting no intertrial variability, the game parameters are always the same, even when the game is repeatedly played.

3C According to Gentile's taxonomy, this exergame (called Worm hurdler) requires stepping tasks. Specifically, the patient represented as an avatar has to overstep a 
crawling worm coming closer alternately from the right and the left side (in-motion regulatory conditions). The parameters of the game are unchangeable and, thus, no intertrial variability is given.

3D The exergame Worm hurdler described in 3C can easily be modified for skill category 3D. By integrating a virtual water jar that has to be balanced on the avatar's head, an object manipulation task is integrated. To avoid water spillage, the patient has to adopt an ideal upright body posture.

4A For skill category 4A, we adapted the game Tractor driver described for category $3 \mathrm{~A}$ by implementing intertrial variability. Specifically, to provide varying regulatory conditions, the hay bale positions change from one exergame trial to another.

4B The exergame Fruit catcher described for skill category 3B needed a slight modification to meet the category requirements for $4 \mathrm{~B}$. In this game, there are not only falling apples which have to be caught, but various kinds of fruits. On the one hand, the fruits have different sizes and, on the other hand, different falling speeds. Obviously, intertrial variability is given.

4C When playing the game Worm hurdler designed for category $3 \mathrm{C}$, but with changing regulatory conditions between the exergame trials, intertrial variability is ensured. Accordingly, in this game, the worms are crawling with varying speeds and are coming either from the right or the left side in random order.

4D For integrating an object manipulation task, the exergame Worm hurdler described for skill category $4 \mathrm{C}$ can be advanced by balancing a virtual water jar on the avatar's head. Consequently, while overstepping the randomly approaching crawling worms, the patient has to adopt an ideal upright body position ensuring that no water is spilling out (from the jar).

\section{Practical application of the taxonomy-based rehabilitation program using exergames}

When implementing a therapeutic program to improve stroke victims motor skills, therapists must select exercises that are tailored to the demands of the individual patient. To achieve optimal outcomes in rehabilitation, the activities should be matched to a patient's functional abilities and limitations. In a well-elaborated training plan, the (exercise) tasks which have to be performed should maintain an optimal challenge for the patient [7, 19]. Obviously, for providing a perfectly tuned exercise difficulty level at any point in time throughout the rehabilitation period, modifying the therapy plan is an essential need and an ongoing process. When a patient is making progress, more challenging tasks should be involved into the rehabilitation program. For selecting functionally appropriate activities during rehabilitation, we highlight the practical value of using Gentile's taxonomy. Based on the consideration that the taxonomy-based skill categories present a structure going from simple to complex motor tasks, we provide a formal procedure for guiding patients from the top left of the table to the bottom right. Closer inspection of Table 1 reveals that the taxonomy can be divided into seven difficulty levels. More specifically, starting from 1 of the 16 skill categories, a category-based progression in task difficulty can be achieved either through a horizontal shift to the right or a vertical shift downward. Accordingly, seven levels of difficulty can be distinguished which define a progressive increase of complexity in diagonal direction (Table 3 ).

Considering the allocation of the skill categories to one of the seven difficulty levels, Gentile's taxonomy offers a simple and easy-to-follow way for incorporating an ongoing progression into a rehabilitation process. Specifically, for selecting appropriate activities that provide rehabilitation at the optimal level of challenge, we propose the following procedure: A patient may only move up one level of difficulty when all skill categories within the current level are completed. For clarification, beginning with skill category $1 \mathrm{~A}$ in difficulty level 1 , the patient is required to move to the skill categories in difficulty level 2 (i.e., $1 \mathrm{~B}$ and $2 \mathrm{~A}$ ) as soon as category $1 \mathrm{~A}$ is successfully performed. Within level 2, patients and treating therapists are free to choose the order of execution of the belonging skill categories. Once a patient is capable to perform both skill categories $1 \mathrm{~B}$ and $2 \mathrm{~A}$ successfully, the next difficulty level 3 has to be incorporated into rehabilitation. This procedure can be continued until the patient finally achieves skill category 4D and, consequently, the highest level of difficulty (i.e., level 7). In this way, challenging situations are ensured at any point in time during rehabilitation and the patients are encouraged to exercise towards their functional limits. Following the procedure suggested to guide someone's therapy plan, one ensures that the selection of skill categories is constrained. Due to this constraint, a variation of both general dimensions defined by Gentile's taxonomy will be considered during the rehabilitation process. Accordingly, the procedure prevents a skill category-based progression only in one direction and includes the modification of both the environmental context and the action function characteristics.

Apart from these constraints, this approach also enables a certain degree of self-determination by therapy-involved actors. Within a specific level of difficulty, the performance order of the skill categories can be freely determined. This autonomy in decision-making increases the potential of tailoring the rehabilitation process to the demands of each individual patient and leaves freedom of choice to the treating therapists for the most adequate exercises that should currently be trained. 
Table 3 Gentile's taxonomy of motor skills divided into seven levels of difficulty (ordered diagonally)

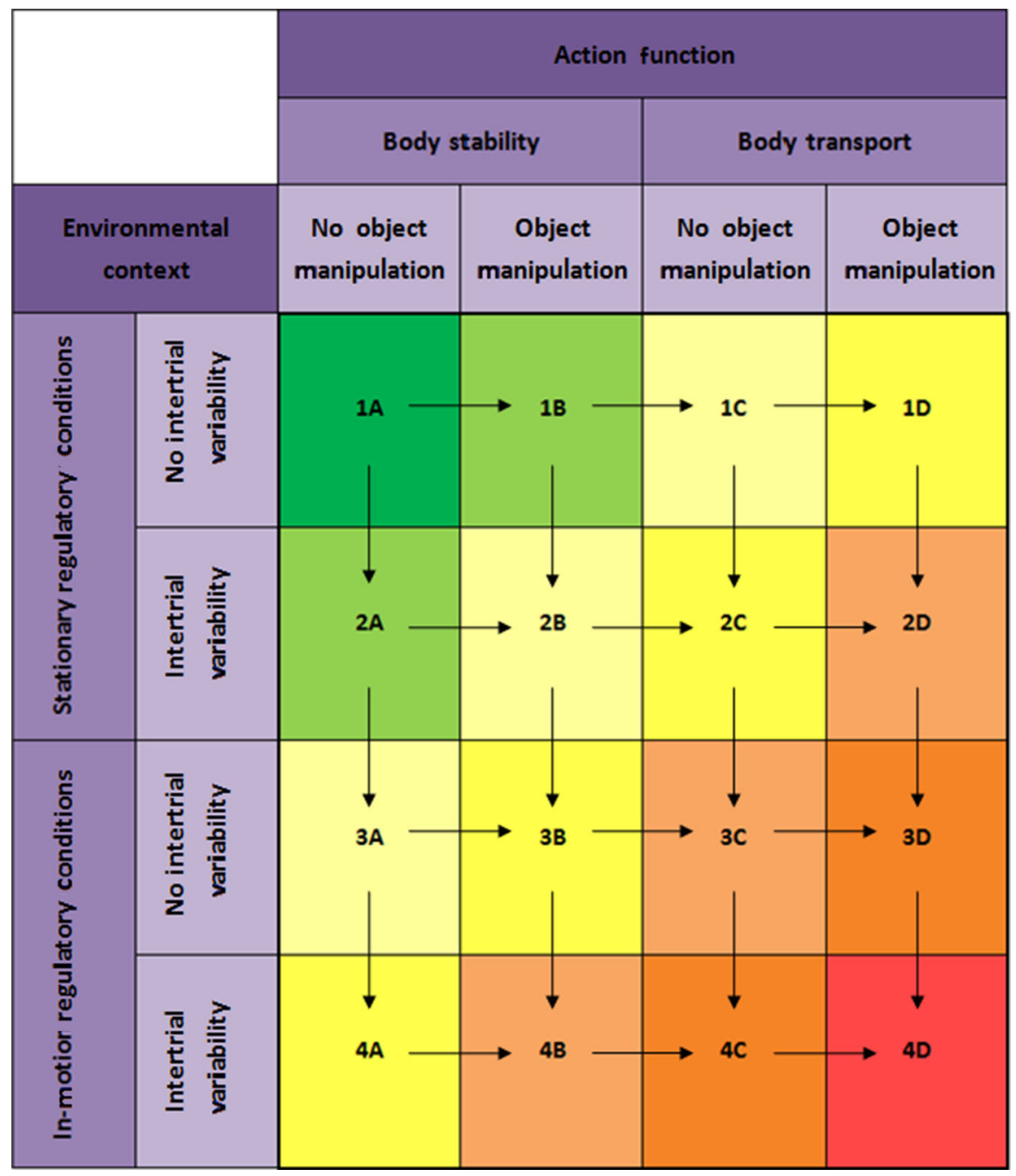

\section{Limitations and future directions}

Using Gentile's taxonomy for designing a rehabilitation program that targets balance and walking deficits of stroke patients, an aspect must be considered critically. According to Gentile's framework, a horizontal shift to the right or a vertical shift downward within the taxonomy provides an increase of task complexity. However, when we focus on a motor task such as standing still on one leg, a potential inconsistency in Gentile's approach emerges. Standing still on one leg can be categorized by the taxonomy dimension body stability that represents less complex tasks than motor skills that require body transport (e.g., walking). In our view, maintaining a one-leg stance position needs good balance skills. Therefore, this activity might be perceived by some stroke patients as an advanced motor task which challenges them more than a body transport activity. Furthermore, where this theoretical approach focused on the "general stroke patient population", we are aware of the fact that different accompanying diseases and impairments; e.g., dizziness or muscle weakness due to stroke, may cause differences in how patients perceive tasks as being more or less difficult. It can be hypothesized that some patients have more difficulty maintaining a given posture without loss of balance whereas others experience performing a body transport task as more difficult. We think, however, that these potential differences may be resolved by clinicians that treat and monitor patients and decide, together with the patients and according their skill levels, how and when to progress through the taxonomy. It is clear to us that several pilot studies assessing feasibility and usability in several reference and subpopulations are needed as necessary next step in our program development process. Feasibility studies are comparative randomized trials designed to provide preliminary evidence on the clinical efficacy of a drug or intervention [55]. Usability evaluation is a way to ensure that interactive systems are adapted to the users and should be part of a fundamental step in the user centered design process [4].

In this paper, we elucidate the theoretical basis for a theorydriven rehabilitation program to improve stroke sufferers' 
balance skills and walking capability using exergames. According to the framework proposed by Campbell et al. [8], investigating relevant theory prior to an exploratory trial is of great importance. Considering the theoretical knowledge gathered, an optimal study intervention design for pilot studies can be worked out that focus on the relevance and effectiveness of the rehabilitation program suggested. Depending on the usability and feasibility testing results obtained, refinements and extensions of the exergames can be made and the present set of six basic exergames may be extended to a maximum of 16 different exergames. Hence, a comprehensive and practical theory-driven stroke rehabilitation program based on Gentile's taxonomy can be achieved.

\section{Conclusion}

With stroke, the changes in the central nervous system and lifestyle may impair an individual's ability to regain and/or maintain certain levels of motor functioning. By using virtual reality, we might be able to offer continued rehabilitation in an attractive and meaningful way. Specific targeted exergames have been designed that aim to minimize stroke-induced walking impairments. Considering the aim of presenting a wellelaborated training concept with a strong theoretical rationale, in this paper we demonstrate that Gentile's taxonomy presents a feasible template providing assistance for developing and implementing a theory-driven rehabilitation program. The motor skill taxonomy suggested by Gentile defines two general dimensions and structures a table that consists of 16 different skill categories (Table 1). On the one hand, based on these skill categories, the taxonomy provides variable training scenarios. Thus, a therapy program which meets the demand of the motor learning principle variable practice can be created. On the other hand, the taxonomy allows a systematic progression during the rehabilitation process. Accordingly, the motor learning principle of progression is explicitly considered. Due to the advantages of Gentile's two-dimensional approach, the taxonomy suits our purposes and was used as underlying framework to build up a post-stroke exergame rehabilitation program for enhancing balance and walking skills. Based on the literature reviewed here, clinical approaches to maintaining and improving physical functioning over longer time periods in persons with stroke may be combined with novel exergame-based approaches that sustain physical functioning.

\footnotetext{
Acknowledgments This work was partially supported by the REWIRE project (www.rewire-project.eu), funded by the European Commission under the FP7 framework with contract 287713.
}

Conflict of Interest Statement Seline Wüest, Rolf van de Langenberg, and Eling D. de Bruin declare that they have no conflict of interest.
Ethical statement This article does not contain any studies with human or animal subjects performed by any of the authors.

Open Access This article is distributed under the terms of the Creative Commons Attribution License which permits any use, distribution, and reproduction in any medium, provided the original author(s) and the source are credited.

\section{References}

1. Adamovich SV, Merians AS, Boian R, Lewis JA, Tremaine M, Burdea GS, Recce M, Poizner H (2005) A virtual reality-based exercise system for hand rehabilitation post-stroke. Presence-Teleop Virt 14(2):161-174. doi:10.1162/1054746053966996

2. Alahakone AU, Senanayake SA (2010) A real-time system with assistive feedback for postural control in rehabilitation. Mechatronics, IEEE/ASME Trans 15(2):226-233

3. Askim T, Indredavik B, Engen A, Roos K, Aas T, Morkved S (2012) Physiotherapy after stroke: to what extent is task-oriented practice a part of conventional treatment after hospital discharge? Physiother Theory Pract. doi:10.3109/09593985.2012.734008

4. Bastien JMC (2010) Usability testing: a review of some methodological and technical aspects of the method. Int J Med Inform 79(4): E18-E23. doi:10.1016/j.ijmedinf.2008.12.004

5. Borghese NA, Pirovano M, Lanzi PL, Wüest S, de Bruin ED (2013) Computational intelligence and game design for effective at-home stroke rehabilitation. Games Health J 2(2):81-88. doi:10.1089/g4h. 2013.0073

6. Burke JW, McNeill MDJ, Charles DK, Morrow PJ, Crosbie JH, McDonough SM (2009) Optimising engagement for stroke rehabilitation using serious games. Visual Comput 25(12):1085-1099. doi: 10.1007/s00371-009-0387-4

7. Cameirao MS, Badia SB, Oller ED, Verschure PF (2010) Neurorehabilitation using the virtual reality based Rehabilitation Gaming System: methodology, design, psychometrics, usability and validation. J Neuroeng Rehabil 7:48. doi:10.1186/1743-0003$7-48$

8. Campbell M, Fitzpatrick R, Haines A, Kinmonth AL, Sandercock P, Spiegelhalter D, Tyrer P (2000) Framework for design and evaluation of complex interventions to improve health. Br Med J 321(7262): 694-696. doi:10.1136/bmj.321.7262.694

9. Carty CP, Cronin NJ, Lichtwark GA, Mills PM, Barrett RS (2012) Mechanisms of adaptation from a multiple to a single step recovery strategy following repeated exposure to forward loss of balance in older adults. PLoS ONE 7(3):e33591

10. Cohen J (1988) Statistical power analysis for the behavioral sciences. Routledge, New York

11. de Bruin ED, Reve EV, Murer K (2013) A randomized controlled pilot study assessing the feasibility of combined motor-cognitive training and its effect on gait characteristics in the elderly. Clin Rehabil 27(3):215-225. doi:10.1177/0269215512453352

12. de Bruin ED, Schoene D, Pichierri G, Smith ST (2010) Use of virtual reality technique for the training of motor control in the elderly. Some theoretical considerations. Z Gerontol Geriatr 43(4):229-234. doi:10. 1007/s00391-010-0124-7

13. Dean CM, Richards CL, Malouin F (2000) Task-related circuit training improves performance of locomotor tasks in chronic stroke: a randomized, controlled pilot trial. Arch Phys Med Rehab 81(4):409417

14. DeJong G, Horn SD, Conroy B, Nichols D, Healton EB (2005) Opening the black box of poststroke rehabilitation: stroke rehabilitation patients, processes, and outcomes. Arch Phys Med Rehab 86(12):S1-S7. doi:10.1016/j.apmr.2005.09.003 
15. Dickstein R, Dunsky A, Marcovitz E (2004) Motor imagery for gait rehabilitation in post-stroke hemiparesis. Phys Ther 84(12):11671177

16. Dombovy ML, Sandok BA, Basford JR (1986) Rehabilitation for stroke - a review. Stroke 17(3):363-369

17. Eliasson AC, Krumlinde-sundholm L, Shaw K, Wang C (2005) Effects of constraint-induced movement therapy in young children with hemiplegic cerebral palsy: an adapted model. Dev Med Child Neurol 47(4):266-275

18. Eng JJ, Tang PF (2007) Gait training strategies to optimize walking ability in people with stroke: a synthesis of the evidence. Expert Rev Neurother 7(10):1417-1436. doi:10.1586/14737175.7.10.1417

19. Fell DW (2004) Progressing therapeutic intervention in patients with neuromuscular disorders: a framework to assist clinical decision making. J Neurol Phys Ther 28(1):35. doi:10.1097/01.NPT. 0000284776.32802.1b

20. Geijtenbeek T, Steenbrink F, Otten B, Even-Zohar O (2011) D-flow: immersive virtual reality and real-time feedback for rehabilitation. In: Proceedings of the 10th International Conference on Virtual Reality Continuum and Its Applications in Industry, Hong Kong. ACM, pp 201-208. doi:10.1145/2087756.2087785

21. Gentile A (2000) Skill acquisition: action, movement, and neuromotor processes. Mov Sci Found Phys Ther Rehabil 2:111-187

22. Guadagnoli MA, Lee TD (2004) Challenge point: a framework for conceptualizing the effects of various practice conditions in motor learning. J Mot Behav 36(2):212-224. doi:10.3200/Jmbr.36.2.212224

23. Hall KG, Magill RA (1995) Variability of practice and contextual interference in motor skill learning. J Mot Behav 27(4):299-309

24. Harley L, Robertson S, Gandy M, Harbert S, Britton D (2011) The design of an interactive stroke rehabilitation gaming system. HumanComput Interact Users Appl Pt Iv 6764:167-173

25. Hatzitaki V, Voudouris D, Nikodelis T, Amiridis I (2009) Visual feedback training improves postural adjustments associated with moving obstacle avoidance in elderly women. Gait Posture 29(2):296-299

26. Hornby TG, Straube DS, Kinnaird CR, Holleran CL, Echauz AJ, Rodriguez KS, Wagner EJ, Narducci EA (2011) Importance of specificity, amount, and intensity of locomotor training to improve ambulatory function in patients poststroke. Top Stroke Rehabil 18(4): 293-307. doi:10.1310/Tsr1804-293

27. Hsu AL, Tang PF, Jan MH (2003) Analysis of impairments influencing gait velocity and asymmetry of hemiplegic patients after mild to moderate stroke. Arch Phys Med Rehabil 84(8):1185-1193

28. Jack D, Boian R, Merians AS, Tremaine M, Burdea GC, Adamovich SV, Recce M, Poizner H (2001) Virtual reality-enhanced stroke rehabilitation. Ieee T Neur Sys Reh 9(3):308-318

29. Kennedy MW, Schmiedeler JP, Crowell CR, Villano M, Striegel AD, Kuitse J (2011) Enhanced feedback in balance rehabilitation using the Nintendo Wii Balance Board. In: e-Health Networking Applications and Services (Healthcom), 2011 13th IEEE International Conference on. IEEE, pp 162-168

30. Kinect for Windows. http://www.microsoft.com/en-us/ kinectforwindows/. Accessed 12 Oct 2013

31. Krakauer JW (2006) Motor learning: its relevance to stroke recovery and neurorehabilitation. Curr Opin Neurol 19(1):84-90

32. Laguna PL (2008) Task complexity and sources of task-related information during the observational learning process. J Sport Sci 26(10):1097-1113. doi:10.1080/02640410801956569

33. Lange B, Flynn S, Rizzo A (2009) Initial usability assessment of offthe-shelf video game consoles for clinical game-based motor rehabilitation. Phys Ther Rev 14(5):355-363

34. Macko RF, Haeuber E, Shaughnessy M, Coleman KL, Boone DA, Smith GV, Silver KH (2002) Microprocessor-based ambulatory activity monitoring in stroke patients. Med Sci Sport Exer 34(3):394-399
35. Magill RA (2004) Motor learning and control: concepts and applications, 7th edn. McGraw-Hill, Boston

36. Merians AS, Jack D, Boiau R, Tremaine M, Burdea GC, Adamovich SV, Recce M, Poizner H (2002) Virtual realityaugmented rehabilitation for patients following stroke. Phys Ther 82(9):898-915

37. Mulder T (1991) A process-oriented model of human motor behavior- toward a theory-based rehabilitation approach. Phys Ther 71(2): $157-164$

38. Muratori LM, Lamberg EM, Quinn L, Duff SV (2013) Applying principles of motor learning and control to upper extremity rehabilitation. J Hand Ther Off J Am Soc Hand Ther 26(2):94-102. doi:10. 1016/j.jht.2012.12.007, quiz 103

39. Muratori LM, Lamberg EM, Quinn L, Duff SV (2013) Applying principles of motor learning and control to upper extremity rehabilitation. J Hand Ther 26(2):94-103

40. Nichols DS (1997) Balance retraining after stroke using force platform biofeedback. Phys Ther 77(5):553-558

41. Nnodim JO, Strasburg D, Nabozny M, Nyquist L, Galecki A, Chen S, Alexander NB (2006) Dynamic balance and stepping versus Tai Chi training to improve balance and stepping in at risk older adults. $\mathrm{J}$ Am Geriatr Soc 54(12):1825-1831

42. O'Dell MW, Lin CCD, Harrison V (2009) Stroke rehabilitation: strategies to enhance motor recovery. Annu Rev Med 60:55-68. doi:10.1146/annurev.med.60.042707.104248

43. Papangelis A, Mouchakis G, Texas AB, Kosmopoulos D, Karkaletsis V, Makedon F (2012) A game system for remote rehabilitation of cerebral palsy patients. In: Proceedings of the 5th International Conference on Pervasive Technologies Related to Assistive Environments. ACM, p 19

44. Pirovano M, Mainetti R, Baud-Bovy G, Lanzi PL, Borghese NA (2012) Self-adaptive games for rehabilitation at home. In: Computational Intelligence and Games (CIG), 2012 I.E. Conference on IEEE. pp 179-186

45. Puh U, Baer G (2009) A comparison of treadmill walking and overground walking in independently ambulant stroke patients: a pilot study. Disabil Rehabil 31(3):202-210. doi:10.1080/ 09638280801903039

46. REWIRE-Rehabilitative Wayout in Responsive Home Environments. http://www.rewire-project.eu/about-rewire. Accessed 22 Feb 2013

47. Richards CL, Malouin F, Wooddauphinee S, Williams JI, Bouchard JP, Brunet D (1993) Task-specific physical therapy for optimization of gait recovery in acute stroke patients. Arch Phys Med Rehab 74(6): 612-620

48. Rizzo A, Kim GJ (2005) A SWOT analysis of the field of virtual reality rehabilitation and therapy. Presence-Teleoperators Virtual Environ 14(2):119-146. doi:10.1162/1054746053967094

49. Salbach NM, Mayo NE, Wood-Dauphinee S, Hanley JA, Richards CL, Cote R (2004) A task-orientated intervention enhances walking distance and speed in the first year post stroke: a randomized controlled trial. Clin Rehabil 18(5):509-519. doi:10.1191/ $0269215504 \mathrm{cr} 763 \mathrm{oa}$

50. Schultheis MT, Rizzo AA (2001) The application of virtual reality technology in rehabilitation. Rehabil Psychol 46(3): 296-311

51. Shaughnessy M, Michael KM, Sorkin JD, Macko RF (2005) Steps after stroke - capturing ambulatory recovery. Stroke 36(6):13051307. doi:10.1161/01.Str.0000166202.00669.D2

52. Shea CH, Kohl RM (1990) Specificity and variability of practice. Res Q Exerc Sport 61(2):169-177

53. Srivastava A, Taly AB, Gupta A, Kumar S, Murali T (2009) Poststroke balance training: role of force platform with visual feedback technique. J Neurol Sci 287(1):89-93 
54. Teixeira-Salmela LF, Olney SJ, Nadeau S, Brouwer B (1999) Muscle strengthening and physical conditioning to reduce impairment and disability in chronic stroke survivors. Arch Phys Med Rehab 80(10): 1211-1218

55. Thabane L, Ma J, Chu R, Cheng J, Ismaila A, Rios LP, Robson R, Thabane M, Giangregorio L, Goldsmith CH (2010) A tutorial on pilot studies: the what, why and how. BMC Med Res Methodol 10:1. doi:10.1186/1471-2288-10-1

56. Tilson JK, Settle SM, Sullivan KJ (2008) Application of evidencebased practice strategies: current trends in walking recovery interventions poststroke. Top Stroke Rehabil 15(3):227-246. doi:10. 1310/Tsrl503-227

57. Tudor-Locke CE, Myers AM, Rodger NW (2001) Development of a theory-based daily activity intervention for individuals with type 2 diabetes. Diabetes Educ 27(1):85-93

58. Tyromotion. http://tyromotion.com/en/products/tymo/overview. Accessed 12 Oct 2013
59. Uzor S, Baillie L (2013) Exploring \& designing tools to enhance falls rehabilitation in the home. In: Proceedings of the SIGCHI Conference on Human Factors in Computing Systems, 2013. ACM, pp 1233-1242

60. Vincent C, Deaudelin I, Robichaud L, Rousseau J, Viscogliosi C, Talbot LR, Desrosiers J (2007) Rehabilitation needs for older adults with stroke living at home: perceptions of four populations. BMC Geriatr 7:20. doi:10.1186/14712318-7-20

61. Vonschroeder HP, Coutts RD, Lyden PD, Billings E, Nickel VL (1995) Gait parameters following stroke - a practical assessment. J Rehabil Res Dev 32(1):25-31

62. Yavuzer G, Eser F, Karakus D, Karaoglan B, Stam HJ (2006) The effects of balance training on gait late after stroke: a randomized controlled trial. Clin Rehabil 20(11):960-969

63. Zyda M (2005) From visual simulation to virtual reality to games. Computer 38(9):25-32 